\title{
Metabolomic profiling implicates adiponectin as mediator of a favorable lipoprotein profile associated with NT-proBNP
}

Annette Masuch ${ }^{1 *+} \mathbb{0}$, Maik Pietzner ${ }^{1,2+}$, Martin Bahls ${ }^{2,3}$, Kathrin Budde ${ }^{1,2}$, Gabi Kastenmüller $^{4}$, Stephanie Zylla ${ }^{1,2}$, Anna Artati ${ }^{5}$, Jerzy Adamski ${ }^{5,6,7}$, Henry Völzke ${ }^{2,8,9}$, Marcus Dörr ${ }^{2,3}$, Stephan B. Felix ${ }^{2,3}$, Matthias Nauck ${ }^{1,2}$ and Nele Friedrich ${ }^{1,2}$

\begin{abstract}
Background: The N-terminal prohormone of brain natriuretic peptide (NT-proBNP) is an important biomarker for the diagnosis of heart failure. Apart from this and only recently recognized, NT-proBNP levels associate with higher HDLand lower LDL-cholesterol levels comprising a favorable blood lipid profile. To further examine this observation, the lipoprotein profile in relation to NT-proBNP was examined in-depth by proton nuclear magnetic resonance spectroscopy ( ${ }^{1} \mathrm{H}$-NMR). We complemented this investigation with a state-of-the-art untargeted metabolomics approach.

Methods: Lipoprotein particles were determined by ${ }^{1} \mathrm{H}$-NMR spectroscopy in 872 subjects without self-reported diabetes from the population-based Study of Health in Pomerania (SHIP)-TREND with available NT-proBNP measurements. Comprehensive metabolomics data for plasma and urine samples were obtained. Linear regression models were performed to assess the associations between serum concentrations of NT-proBNP and the metabolites/lipoprotein particles measured in plasma or urine.

Results: An increase in serum NT-proBNP was associated with a benefical lipoprotein profile, including a decrease in VLDL, IDL and LDL-particles along with an increase in large HDL particles. These findings were replicated in a second independent cohort. Serum concentrations of NT-proBNP showed significant inverse associations with seven plasma metabolites while associations with 39 urinary metabolites, mostly comprising amino acids and related intermediates, were identified. Mediation analyses revealed adiponection as mediating factor for the associations observed with lipoproteins particles.

Conclusions: Most of the metabolic changes associated with NT-proBNP implicate significant influence on the blood lipid profile besides vasodilatory and the diuretic action of BNP signaling. Our data suggest that the more favorable lipoprotein profile as associated with elevated NT-proBNP concentrations in mainly cardiac healthy individuals might relate to adiponectin signaling indicating even indirect cardio-protective effects for NT-proBNP.
\end{abstract}

Keywords: Natriuretic peptides, Metabolomics, Adiponectin, Lipoproteins, Population-based study

\footnotetext{
*Correspondence: annette.masuch@uni-greifswald.de

${ }^{\dagger}$ Annette Masuch and Maik Pietzner contributed equally to this work

${ }^{1}$ Institute of Clinical Chemistry and Laboratory Medicine, University

Medicine Greifswald, Ferdinand-Sauerbruch-Str, 17475 Greifswald,

Germany

Full list of author information is available at the end of the article
}

(c) The Author(s) 2018. This article is distributed under the terms of the Creative Commons Attribution 4.0 International License (http://creativecommons.org/licenses/by/4.0/), which permits unrestricted use, distribution, and reproduction in any medium, provided you give appropriate credit to the original author(s) and the source, provide a link to the Creative Commons license, and indicate if changes were made. The Creative Commons Public Domain Dedication waiver (http://creativecommons.org/ publicdomain/zero/1.0/) applies to the data made available in this article, unless otherwise stated. 


\section{Introduction}

The $\mathrm{N}$-terminal fragment of the prohormone of brain natriuretic peptide (NT-proBNP) plays a central role as a biomarker in the diagnosis of heart failure (HF), a syndrome resulting in reduced cardiac output and/ or elevated intracardiac pressure at rest or during stress [1]. Elevated levels of NT-proBNP are correlated with increased ventricular mass, another hallmark of chronic HF, and identify pulmonary hypertension [2]. NT-proBNP is the biologically inactive product resulting from the cleavage process which deliberates and activates BNP from proBNP $[3,4]$. BNP is produced and released in response to distension or wall stress of the heart and is known to have essential functions in water balance and blood pressure by inducing vasodilation and thereby acting antagonistically to angiotensin II. Further, BNP increases endothelial permeability, directly inhibits aldosterone synthesis and the release of renin, and induces diuresis and natriuresis in the kidney [3, 5]. Recent evidence indicates that natriuretic peptides possess functions beyond cardiovascular homeostasis and relates them to the function and development of adipose tissue [6,7]. In line with the assumption of an interrelation between body fat and the natriuretic hormone system, higher BNP levels within the reference range were associated with a favorable distribution of adipose tissue meaning lower visceral and liver fat and increased lower-body fat in participants of the DALLAS Heart Study [8]. NTproBNP and BNP levels are inversely correlated with $\mathrm{BMI}$ in the general population and obese individuals have typically lower BNP concentrations independent of cardiovascular disease (CVD) $[9,10]$. A recent study further revealed that BNP within the reference range $(<100 \mathrm{ng} / \mathrm{L})$ was inversely correlated with total cholesterol, non-HDL cholesterol, and non-fasting triglycerides representing a favorable lipoprotein profile confirming an observation from a number of previous studies [11-13]. In contrast, in CVD-for which dyslipidemia is a well-known risk factor [14] -NT-proBNP or BNP levels are typically elevated. Importantly, the relation between NT-proBNP and the lipoprotein profile was also observable for concentrations above the reference limit and in the very elderly $[15,16]$.

In the disease setting, increased NT-proBNP or BNP levels reflect the attempt of the body to restore homeostasis in response to increased intracardiac pressure and/ or reduced cardiac output. Thus, BNP is elevated secondary to cardiac dysfunction and independent of adipose tissue function. Overall, NT-proBNP is involved in very different and independent physiologic processes including cardiovascular homeostasis and adipose tissue function.
In order to improve the understanding of NTproBNP beyond cardiac dysfunction and to explore the relation to lipoprotein metabolism, the present study aimed to examine the associations of NT-proBNP plasma concentrations with the metabolic profile of a large population. As previous studies on NT-proBNP found adiponectin to be associated in a similar manner with a favorable lipoprotein profile $[17,18]$, a mediation analysis was performed. For this purpose, in-depth analyses of the blood lipid profile associated with NTproBNP were done using highly resolved lipoprotein particle measures obtained by proton nuclear magnetic resonance $\left({ }^{1} \mathrm{H}-\mathrm{NMR}\right)$ spectroscopy. In order to further broaden the view on possible metabolic implications of NT-proBNP concentrations we complemented these analyses with an untargeted metabolomics approach based on mass spectrometry (MS) and ${ }^{1} \mathrm{H}$-NMR spectroscopy using plasma and urine samples from the same individuals.

\section{Materials and methods \\ Study population-discovery cohort}

The Study of Health in Pomerania (SHIP-TREND) is a population-based study located in West Pomerania, a rural region in north-east Germany [19]. A stratified (age, sex and city/county of residence) random sample of 8826 adults aged $20-79$ years was drawn from population registries. Sample selection was facilitated by centralization of local population registries in the Federal State of Mecklenburg-West Pomerania. Baseline examinations were conducted between 2008 and 2012. In total, 4420 subjects chose to participate (50.1\% response).

For a subsample of 1000 subjects without self-reported diabetes plasma and urine metabolomics data based on MS and ${ }^{1} \mathrm{H}-\mathrm{NMR}$ were available. After excluding subjects with missing values in serum NT-proBNP measurements, confounding variables or echocardiography the final study sample comprised 872 individuals. Please see below for specification of variables and confounders (Methods, Statistical analysis).

\section{Study population-replication cohort}

Associations between NT-proBNP and lipoprotein particle data obtained by ${ }^{1} \mathrm{H}-\mathrm{NMR}$ spectroscopy were replicated in the 10 year follow-up of the independent SHIP cohort (SHIP-2) [19]. After application of the same exclusion criteria as outlined above 1420 out of 2333 participants were available for replication. A brief description of the sampling procedure and the study population is given in the Supplemental Information (Additional file 1: Table S1). 


\section{Ethics, consent and permissions}

All participants gave written informed consent before taking part in the study. The study was approved by the local ethics committee and conformed to the principles of the declaration of Helsinki. SHIP data are publically available for scientific and quality control purposes. Data usage can be applied for via http://www.communitymedicine.de.

\section{Laboratory measurements and phenotypic characterization}

Smoking status (current, former or never smokers), daily alcohol consumption and physical activity ( $\geq 1 \mathrm{~h}$ training a week) were assessed using computer-aided personal interviews. Waist circumference (WC) was measured to the nearest $0.1 \mathrm{~cm}$ using an inelastic tape midway between the lower rib margin and the iliac crest in the horizontal plane.

Fasting blood samples were taken from the cubital vein of participants in the supine position between 7.00 and $13.00 \mathrm{~h}$. In the same time span spot urine samples were taken. All samples were either analyzed immediately or stored at $-80{ }^{\circ} \mathrm{C}$. Blood lipids (total cholesterol, high-density [HDL] and low-density lipoprotein [LDL] cholesterol, triglycerides), serum enzymatic activity concentration of alanine amino transferase (ALT) and serum NT-proBNP were measured by standard methods (Dimension VISTA, Siemens Healthcare Diagnostics, Eschborn, Germany). Serum adiponectin levels were determined using an enzyme-linked immunosorbent assay (Mediagnost, Germany). The inter-assay coefficients of variation were $6.75 \%$ and $6.18 \%$ for low and high concentrations, respectively. With respect to NTproBNP, the coefficients of variation were $2.5 \%$ at low, $2.3 \%$ at medium, and $3.6 \%$ at high concentrations of control material, respectively.

Serum creatinine was measured using an enzymatic assay (Dimension VISTA, Siemens Healthcare Diagnostics, Eschborn, Germany) and subsequently the estimated glomerular filtration rate (eGFR) was calculated using the CKD-EPI equation [20].

\section{Echocardiography}

Two-dimensional, M-Mode and Doppler echocardiography were performed using the Vingmed CFM 800A system (GE Medical Systems, Waukesha, USA) as described in detail elsewhere [21]. Measurements of LV end-diastolic and end-systolic diameter (LVD, LVS) were performed according to the guidelines of the American Society of Echocardiography [22]. LV ejection fraction (EF) were calculated following the formula below according to the guidelines of the American Society of Echocardiography [23]: EF (\%) = (LVDV - LVSV)/LVDV.

\section{Metabolomics measurements}

A detailed description of all applied measurement techniques is given in the Additional file 1. Briefly, four different approaches were combined in the discovery cohort: (1) non-targeted MS-based profiling of plasma and urine samples as reported previously [24] (2) targeted MSbased profiling of plasma samples using the AbsoluteIDQ p180 Kit (BIOCRATES LifeSciences AG, Innsbruck, Austria) (3) ${ }^{1} \mathrm{H}$-NMR-based profiling of urine samples as reported previously [25] and (4) ${ }^{1} \mathrm{H}$-NMR-based profiling of plasma samples to derive lipoprotein particles.

After quality control and pre-processing (see Additional file 1) 613 plasma and 587 urine metabolites were available for statistical analyses. Note that some of these could not be unambiguously assigned to a chemical identity and are referred to hereafter with the notation " $\mathrm{X}$ " followed by a unique number. Data on lipoprotein particles comprise 117 measures describing the gradient from very-low-density lipoprotein (VLDL) particles to HDL particles, including their triglycerides, cholesterol, free cholesterol, phospholipid as well as apolipoprotein $B$ (ApoB), A1 (Apo-A1) and A2 (Apo-A2) content.

Metabolomics data in SHIP-2, i.e. the replication cohort, were restricted to ${ }^{1} \mathrm{H}-\mathrm{NMR}$-based profiling of plasma samples with the same approach as described above for SHIP-TREND.

\section{Statistical analyses}

Continuous data are expressed as median (25th; 75th quartile) and nominal data are expressed as percentage. For bivariate analyses, the Kruskal-Wallis test (continuous data) or $\chi^{2}$ test (nominal data) was used to compare women and men. Linear regression models were performed to assess the associations between serum concentrations of NT-proBNP and the metabolites measured in plasma and urine (dependent variables). To stabilize variances in linear regression analyses NT-proBNP concentrations and metabolite levels were log-transformed yielding a clear improvement towards normality. Adjustment in linear regression analysis comprised age, sex, WC, smoking, physical activity, eGFR, and ALT levels. A possible modifying effect of sex was tested by inclusion of an interaction term in the linear model. Further, sensitivity analyses were done excluding subjects who reported intake of lipid lowering medication (ATC code C10; $\mathrm{N}=59$ ). Possible non-linear associations between NTproBNP and metabolites/lipoproteins, e.g. achievement of a plateau phase, were tested by means of restricted cubic splines with three knots [26]. To account for multiple testing, we adjusted the p-values from regression analysis by controlling the false discovery rate (FDR) at $5 \%$ using the Benjamini-Hochberg procedure. Integration of multi-fluid data was achieved by computation of 
metabolic networks using Gaussian graphical modelling (GGM). The procedure is outlined in the Additional file 1.

As literature search suggested a possible mediating effect of adiponectin on the association between NT-proBNP and lipoprotein measures, we performed mediation analyses as implemented in the $R$ package mediate. We defined a significant mediation if the $\mathrm{p}$ value was $<0.01$ (using 2000 bootstrap samples) and at least $10 \%$ of the association was mediated. Statistical analyses were performed using SAS version 9.4 (SAS statistical software, version 9.4, SAS Institute, Inc; NC, USA) and R 3.1.1 (R Foundation for statistical computing, version 3.1.1, Vienna, Austria).

\section{Results}

\section{Study population}

Age, eGFR, and physical activity were comparable between men and women (Table 1). However, women showed a more benficial lipid profile, mainly due to higher HDL-cholesterol values, compared to men. NTproBNP levels were clearly higher in women despite having a slightly better median ejection fraction.

As no obvious sex-interactions $(\mathrm{FDR}<0.2)$ were observed we did not stratify the analyses despite the strong differences in NT-proBNP concentrations between men and women.

\section{Plasma lipoproteins}

Figure 1 presents the results of NT-proBNP concentrations associated with the whole range of plasma lipoprotein particles and contents. An increase in serum NT-proBNP was associated with a beneficial lipoprotein profile, including less VLDL, intermediate-density lipoprotein (IDL), and LDL-particles along with higher large HDL particle measures and lower small dense HDL particle measures (Fig. 1). Even plasma cholesterol and triglycerides were significantly inversely associated with NT-proBNP. In particular, an inverse association with measures of small dense LDL-particles was observable. The ratio between total Apo-B and Apo-A1 reflected the beneficial shift in the lipid profile with higher NTproBNP as well (Fig. 1).

The exclusion of subjects taking lipid-lowering medication did not lead to any substantial changes in the association results with respect to the lipoprotein profile (Additional file 1: Fig. S1). Further, with the exception of the association with HDL2-particles, all associations could be replicated in the independent SHIP-2 cohort (Additional file 1: Fig. S2).
Table 1 General characteristics of the study population

\begin{tabular}{|c|c|c|c|}
\hline Characteristic & Men $(n=373)$ & Women $(n=499)$ & $\mathrm{p}^{*}$ \\
\hline Age (years) & $49(38 ; 60)$ & $49(40 ; 59)$ & 0.94 \\
\hline Smoking (\%) & & & $<0.01$ \\
\hline Never smoker & 32.7 & 49.7 & \\
\hline Former smoker & 44.8 & 28.5 & \\
\hline Current smoker & 25.5 & 21.8 & \\
\hline Physically active (\%) & 74.3 & 73.7 & 0.93 \\
\hline $\begin{array}{l}\text { Waist circumference } \\
\quad(\mathrm{cm})\end{array}$ & $93(85 ; 101)$ & $80(73 ; 89)$ & $<0.01$ \\
\hline NT-probnp (ng/L) & $32(19 ; 55)$ & $68(41 ; 124)$ & $<0.01$ \\
\hline Triglycerides (mmol/L) & $1.27(0.92 ; 1.88)$ & $1.13(0.81 ; 1.60)$ & $<0.01$ \\
\hline $\begin{array}{l}\text { HDL-cholesterol } \\
\text { (mmol/L) }\end{array}$ & $1.29(1.11 ; 1.49)$ & $1.58(1.35 ; 1.83)$ & $<0.01$ \\
\hline $\begin{array}{l}\text { LDL-cholesterol } \\
\text { (mmol/L) }\end{array}$ & $3.35(2.73 ; 3.96)$ & $3.31(2.72 ; 3.95)$ & 0.55 \\
\hline $\begin{array}{l}\text { Total cholesterol } \\
(\mathrm{mmol} / \mathrm{L})\end{array}$ & $5.3(4.5 ; 6.0)$ & $5.5(4.9 ; 6.2)$ & $<0.01$ \\
\hline Egfr $\left(\mathrm{mL} / \mathrm{min} / 1.73 \mathrm{~m}^{2}\right)$ & $98(89 ; 108)$ & $98(86 ; 107)$ & 0.28 \\
\hline $\mathrm{ALT}(\mu k \mathrm{katal} / \mathrm{L})$ & $0.46(0.35 ; 0.64)$ & $0.30(0.24 ; 0.41)$ & $<0.01$ \\
\hline Ejection fraction (\%) & $72(65 ; 77)$ & $73(68 ; 79)$ & $<0.01$ \\
\hline Self-reported HF (\%) & $1.9 \%$ & $2 \%$ & 1.00 \\
\hline Adiponectin (ng/mL) & $5644(3814 ; 7782)$ & $8851(6333 ; 12,102)$ & $<0.01$ \\
\hline
\end{tabular}

Continuous data are expressed as median (25th percentile; 75 th percentile); nominal data are given as percentages. ${ }^{*} x^{2}$-test (nominal data) or MannWhitney-U test (interval data) were performed to compare men and women $H D L$ high density lipoprotein, $L D L$ low-density lipoprotein, NT-proBNP N-terminal prohormone of brain natriuretic peptide, eGFR estimated glomerular filtration rate, $A L T$ alanine aminotransferase, $H F$ heart failure

\section{Plasma and urine metabolome}

Serum concentrations of NT-proBNP showed solely significant inverse associations with seven plasma metabolites (Fig. 2). Those included androsterone sulfate, glycerophosphocholine (GPC), the diacyl phosphatidylcholine (PC aa XX:Y) PC aaC40:5, the lysophosphatidylcholine (lysoPC) C18:0, uridine and the two unknown compounds X-12844 and X-11444.

A total of 39 urine metabolites showed significant associations with NTproBNP (Fig. 2). One signature comprised amino acid species, in particular inverse associations with aromatic and branched-chain amino acids (BCAA) and related degradation intermediates (Fig. 2). Only C-mannosyltryptophan, 3-methylglutaconate, and 1-methylimidazoleacetate were an exception showing positive associations. Further inverse associations were observed for urinary levels of 11-ketoetiocholanolone glucuronide, glycolate, 1-methylxanthine, and methanol as well as several unknown compounds. Among the latter, the strong inverse associations with X-11444 and X-12844 seen in plasma were replicated in urine (Fig. 2). Positive associations with serum NT-proBNP comprised neopterin, $N$-acetylneuraminate, pseudouridine, adenosine, phosphate, and acetone as well as several unknown compounds. 


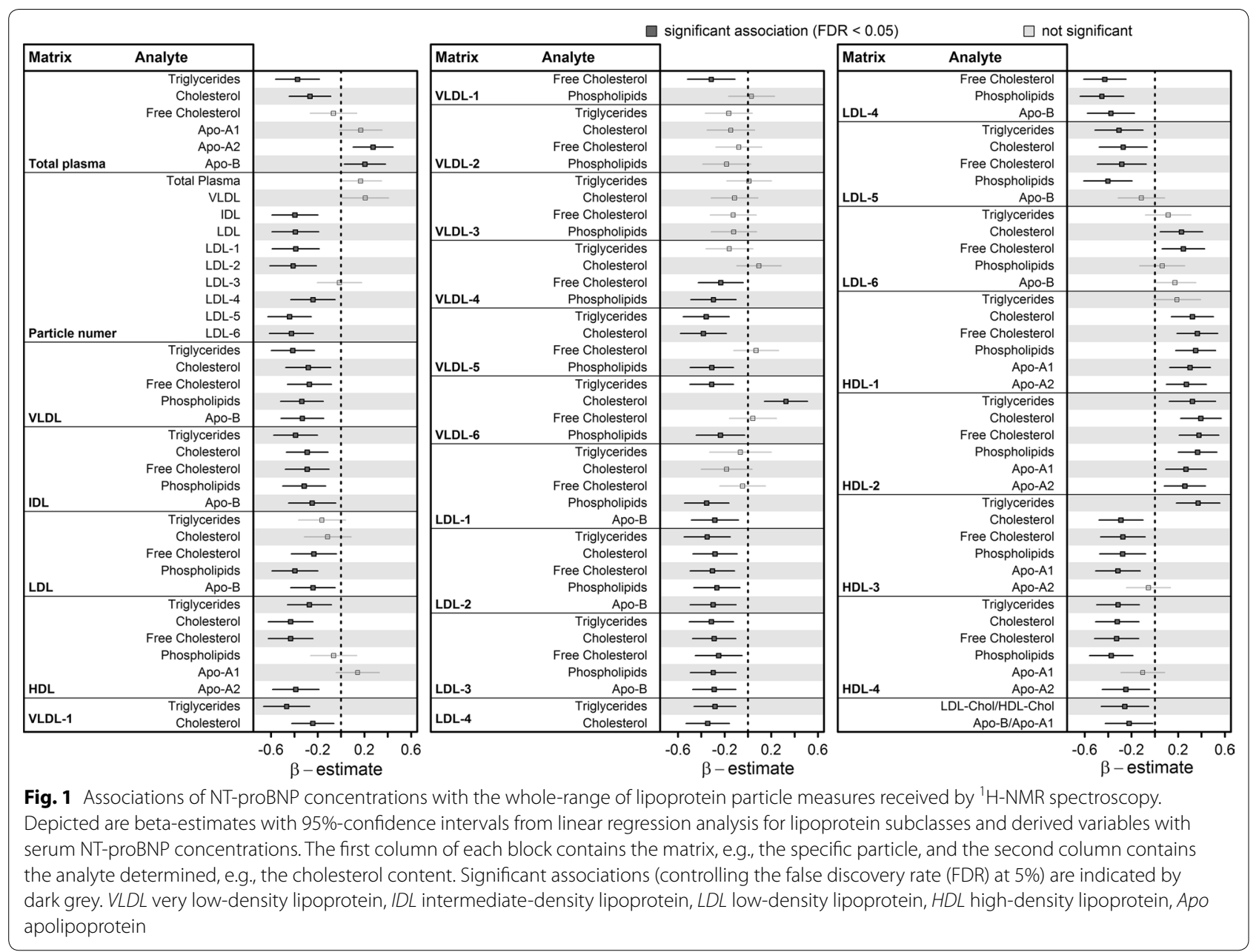

\section{Integration of multifluid data and unknown parameters}

To integrate urine and plasma metabolome data GGMs were computed to reconstruct physiological dependencies in a data driven manner. After discarding metabolites with more than $20 \%$ missing values, the final GGM consisted of 785 nodes and 1065 edges. Subsequently, we projected the results, i.e. beta estimates and p-values, from linear regression analyses on the metabolite network by increasing node size (as $-\log 10$ (p-value)) and color gradient (beta estimate ranging from blue, strong inverse association, to orange, strong positive association). Direct neighborhood within the GGM is a strong indicator of biochemical, and hence physiological, dependencies between metabolites [27]. By visual inspection of such a modified GGM we could integrate the unknown metabolites X-11444 and 12844, which were strongly associated with NT-proBNP in both fluids, together with 11-ketoetiocholanolone glucuronide into a steroid metabolite cascade (Fig. 3).

\section{Mediation by serum adiponectin concentrations}

Plasma adiponectin showed a significant correlation with serum NT-proBNP $(r=0.30 ; p<0.01 ;$ Fig. 4). Mediation analyses revealed a sustainable influence of serum adiponectin on almost all associations between serum NT-proBNP and lipoprotein subclass measures (Fig. 4) reaching a median proportion mediated of about 0.2 . In particular, associations with VLDL, small dense LDL as well as large HDL particles were affected. The association with total cholesterol was not affected. Notably, with the exception of the urine unknown X-17340 none of the small molecule associations was affected.

\section{Discussion}

The present study examined the metabolic fingerprint of NT-proBNP in a sample from the general population without self-reported diabetes to decipher underlying metabolic reasons for the observed associations of higher NT-proBNP concentrations with the presence 


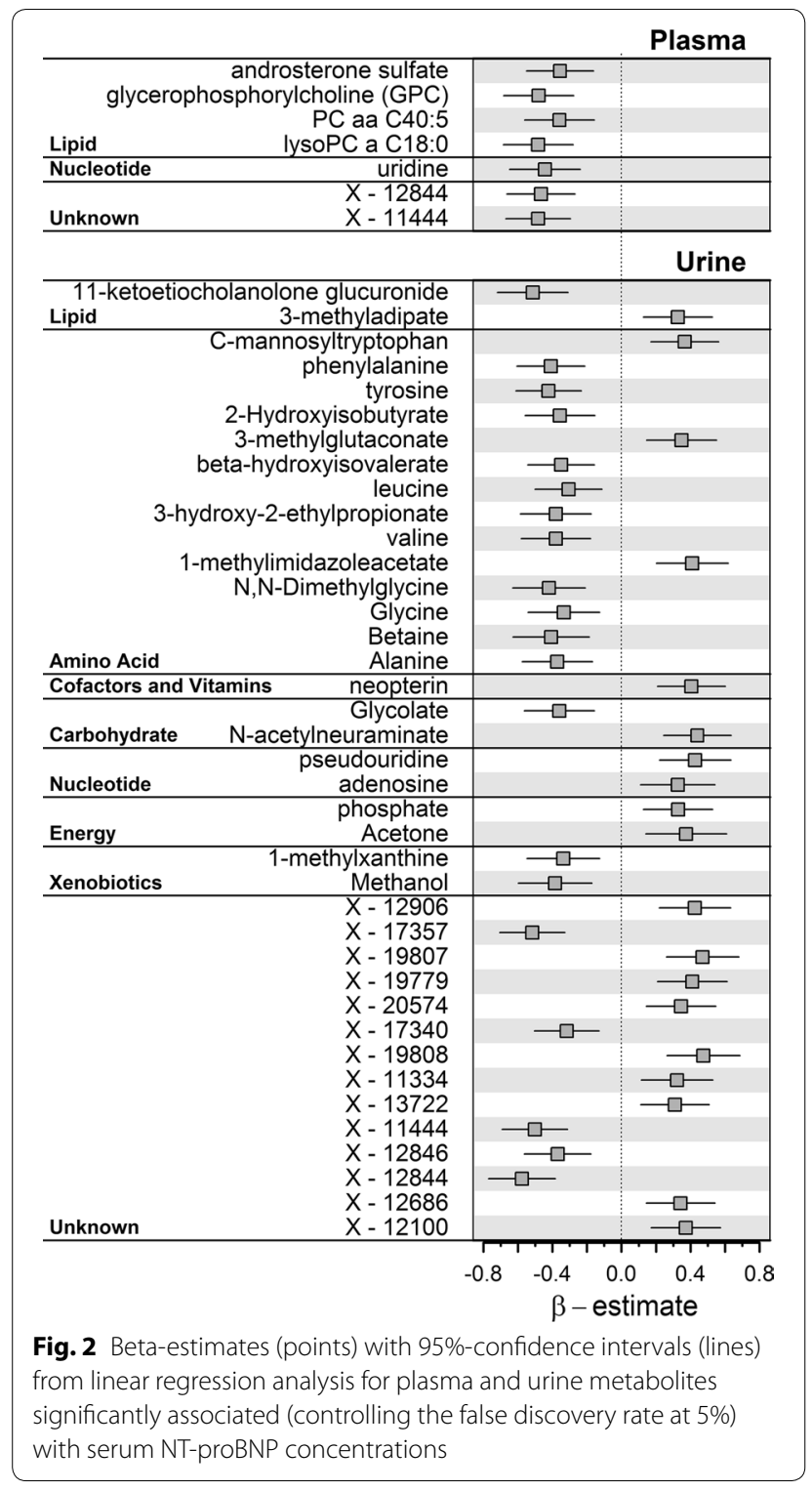

of a beneficial lipoprotein profile. Almost the whole density gradient of the lipoprotein profile was positively shifted which was observed in two independent cohorts. Using a non-targeted metabolomics approach revealed only few metabolites in plasma but several metabolites in urine to be associated with NT-proBNP.

For the comprehensive understanding of NT-proBNP associated metabolites, the physiologic role of BNP as the biologically active mature peptide after cleavage from NT-proBNP has to be considered. NT-proBNP and BNP are released in equimolar amounts but have different plasma half-life with $120 \mathrm{~min}$ and $20 \mathrm{~min}$, respectively [28]. BNP as a member of the natriuretic peptide hormone family modulates diverse biological functions, including regulation of blood pressure and water balance, which were partially reflected by the metabolic signature seen with respect to NT-proBNP. As mainly cardiac healthy individuals were examined, the observed associations can be considered independent of severe cardiac dysfunction. Importantly, the present results indicate that the relation between NTproBNP and a beneficial lipoprotein profile might be mediated by adiponectin.

\section{Beneficial lipoprotein profile}

In general, low HDL-cholesterol and high LDL-cholesterol are significantly associated with the risk to develop CVD [29]. Notably, besides being a marker of cardiac dysfunction, higher values of NT-proBNP were associated with a more beneficial lipoprotein profile among our apparently healthy study population as well as in a population of more advanced age (SHIP-2). Our findings are in line with an observation among 680 elderly volunteers with NT-proBNP levels below $100 \mathrm{ng} / \mathrm{L}$ exhibiting an inverse association between BNP and non-HDL cholesterol [11]. Likewise, Takeuchi et al. observed a strong inverse correlation between serum BNP concentrations and total cholesterol in a population of patients [30]. In older adults free of cardiovascular or kidney disease increased levels of NT-proBNP related to lower LDL-cholesterol and higher HDL-cholesterol [17]. Notably, adiponectin levels were similarly associated with a favorable lipoprotein profile in this study, which was also reported by others [18]. Also serum adiponectin was linked to serum (NT-pro)BNP [18, 31]. Indeed, with respect to a possible mechanism accounting for these observations, the mediation of the association of NT-proBNP with a beneficial lipoprotein profile via adiponectin has been shown in the present study.

\section{Adiponectin in the cardiovascular system}

Adiponectin is an adipokine which is known to be almost exclusively produced and secreted by adipocytes. However, in addition to adipocytes, various other cell types and tissues secrete adiponectin. Besides its main metabolic role in glucose homeostasis, adiponectin is also implicated in many other metabolic and inflammatory functions, including for instance tissue regeneration [32] and proliferation and function of sebocytes [33]. Considering a relation between BNP and adiponectin, it has to be noted that perivascular adipose tissue (PVAT) has significant influence on the vascular tone releasing anti-contractile factors, one of them being adiponectin. The overall function of PVAT with respect to vasodilation seems to be strongly dependent on cGMP-dependent protein kinase (PKG) [34] which is also the effector kinase activated in response to $\mathrm{BNP}$-binding to the natriuretic peptide 


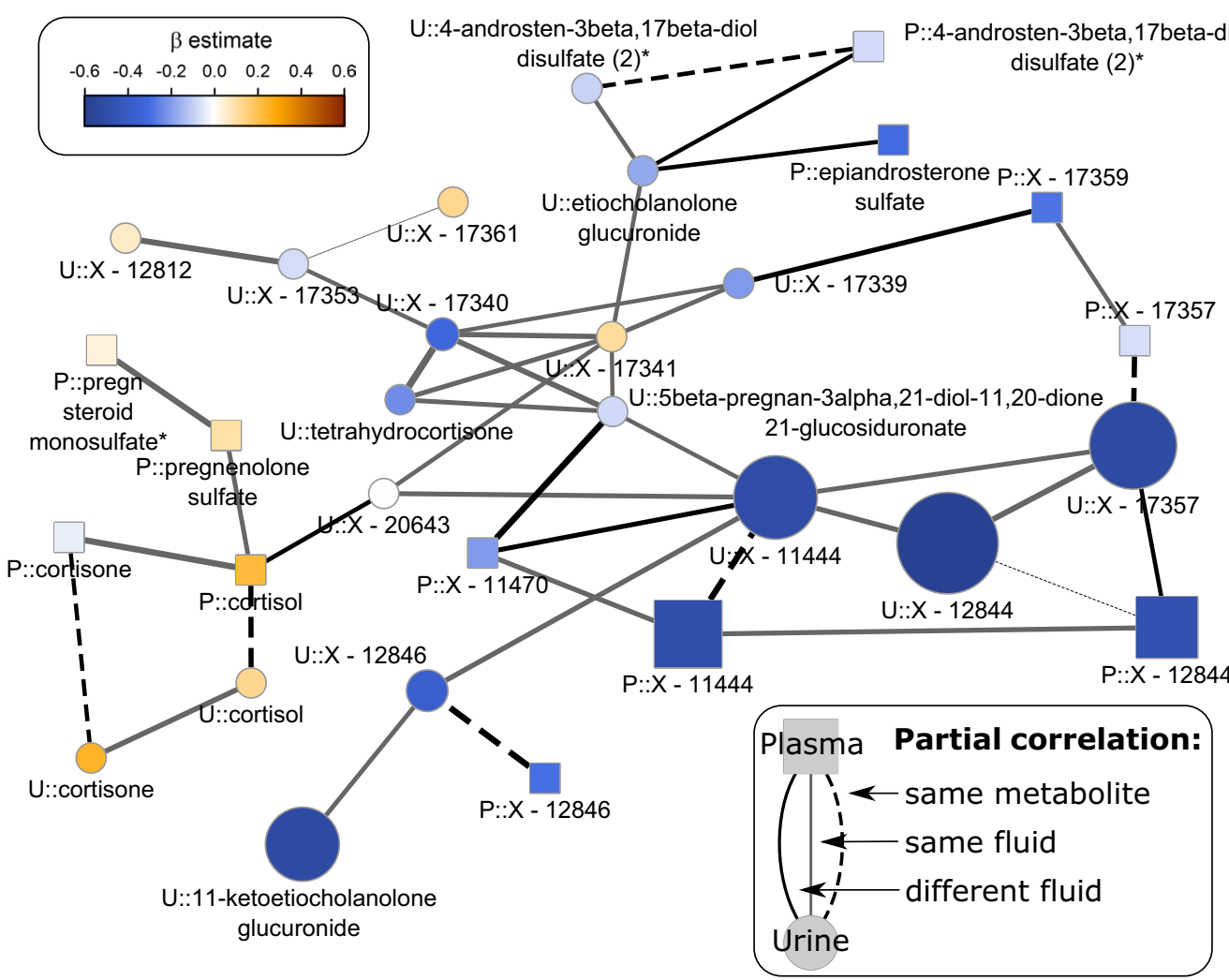

Fig. 3 Subnetwork from the Gaussian graphical model to reconstruct metabolite dependencies with a particular focus on adrenal steroids. Node size is determined by - $\log 10$ (FDR-value) from linear regression analyses with NT-proBNP as exposure and colors indicate effect directionsblue $=$ inverse and orange $=$ positive. Suffix P:: and U:: indicate plasma and urine metabolites, respectively
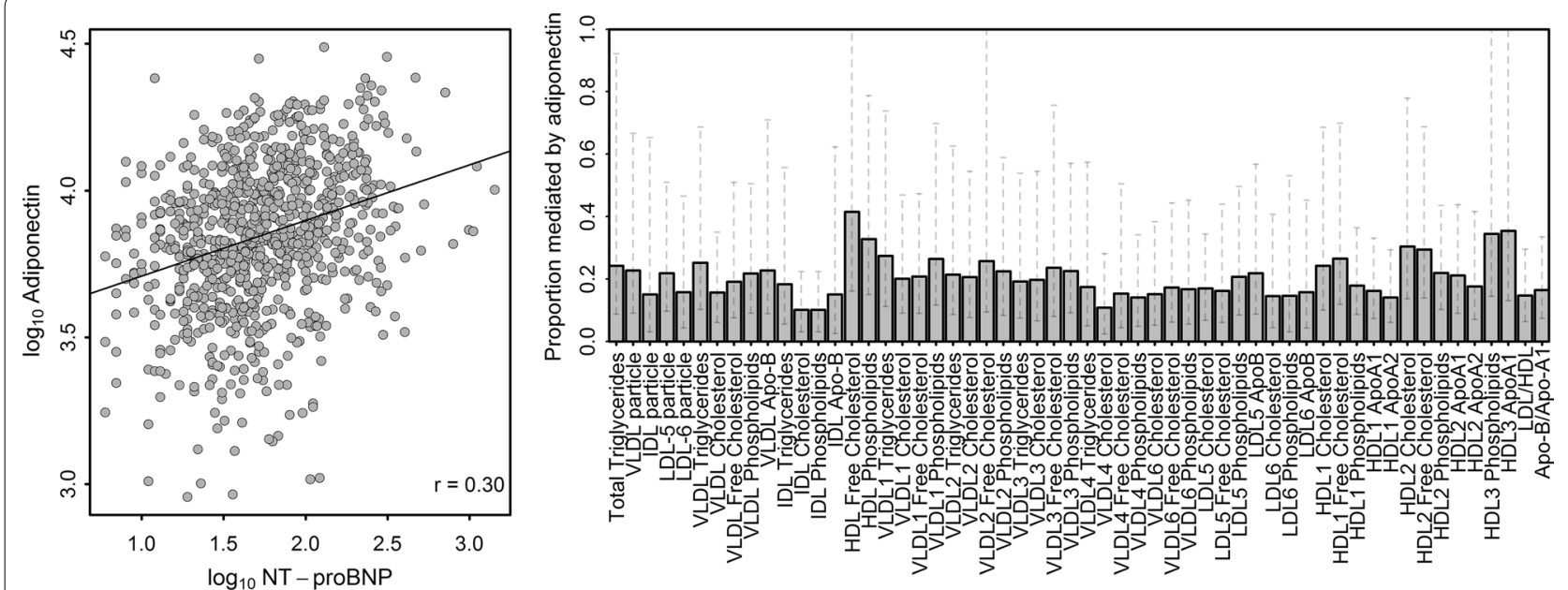

Fig. 4 Left: Scatterplot of serum N-terminal prohormone of brain natriuretic peptide (NT-proBNP) and adiponectin concentrations on a

logarithmized scale. The black line indicates a simple linear regression fit. Right: Proportion mediated (bars) with $95 \%$ confidence intervals by serum adiponectin concentrations for lipoprotein subclass measures significantly associated with serum NT-proBNP in linear regression analyses 
receptor A (NPR-A) [2]. In view of these complementary vasodilator effects described for of BNP and PVAT and comprising both the activation of PKG, it is tempting to assume that increased BNP may directly relate to enhanced adiponectin release from adipocytes. Indeed, Tsukamoto et al. showed in cultured human adipocytes increased adiponectin gene expression and adiponectin secretion in response to natriuretic peptide treatment and the study also demonstrated increased plasma levels of adiponectin in patients with congestive HF receiving atrial natriuretic peptide [35]. Adiponectin in turn alters lipoprotein metabolism on different levels, e.g. by suppressing hepatic lipase activity [36] or promoting activation of lipoprotein lipase [37, 38]. Taken together, a potential pathway that may contribute to the improved lipoprotein profile associated with NTproBNP might rely on BNP signaling on adipocytes and NPR-A activation promoting PKG elevation followed by enhanced adiponectin release which finally influences lipoprotein metabolism (Fig. 5).

Numerous studies uncovered various functions of adiponectin which further support its positive metabolic influence. Oxidative stress plays a pivotal role in inflammatory processes and in turn contributes to development and progression of a number of diseases including CVD, type 2 diabetes mellitus (T2DM) and neurodegenerative diseases [39]. In this context, adiponectin has been reported to reduce production of reactive oxygen species preventing damage due to free radicals [40]. In line with this, in patients with T2DM levels of circulating adiponectin was lower in serum compared to normoglycemic subjects and vascular free radical formation related to NADPH oxidase was increased, which in turn activated PVAT to increase adiponectin expression in response to increased free radical formation [41]. Higher circulating adiponectin levels were also associated with a beneficial development of heart rate variability over 5 years in T2DM representing improved cardiovascular autonomic function [42]. Comparing a native Japanese population with Japanese-American people revealed lower serum adiponectin and increased measures of inflammation in the later indicating that westernization of lifestyle may affect adiponectin which in turn might promote development of insulin resistance [43]. Strengthening the role of adiponectin in T2DM, an increase of circulating adiponectin in response to anti-diabetic treatment has been reported [44]. However and in contrast to these reports, adiponectin has been found to be positively associated with increased cardiovascular mortality rate. The reasons for this observations remain to be unraveled [45].

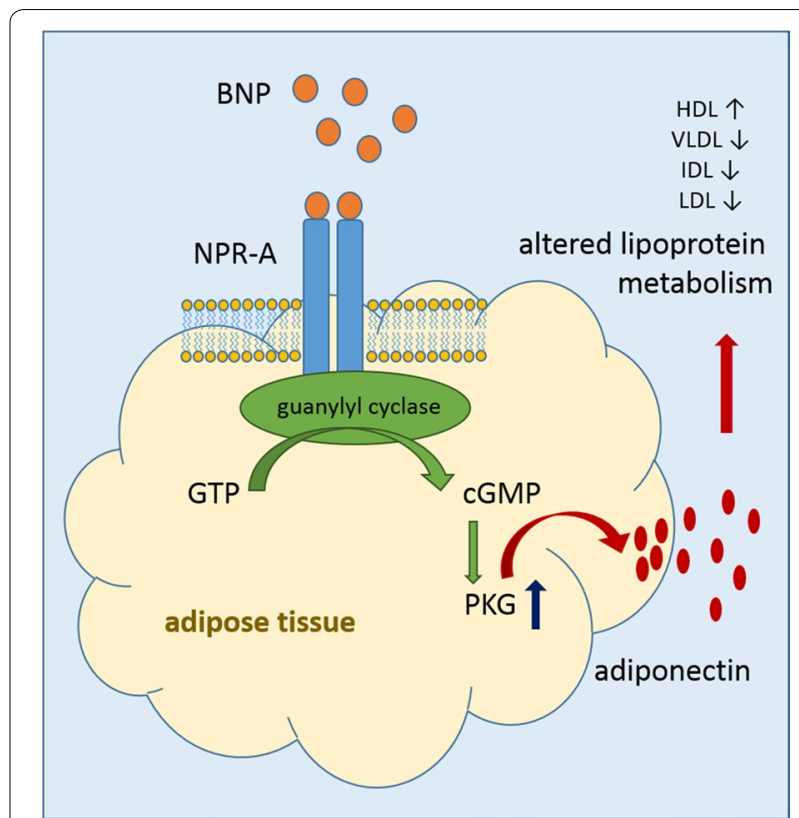

Fig. 5 Proposed pathway based on the analysis of the metabolome and lipidome data. BNP binds to NPR-A in adipose tissues leading to activation of guanylyl cyclase catalyzing the formation of cGMP from GTP. PKG is activated promoting the release of adiponectin which in turn affects lipoprotein metabolism shifting the lipoprotein profile towards a more favorable status. BNP brain natriuretic peptide; NPR-A natriuretic peptide receptor A, GTP guanosine triphosphate, cGMP cyclic guanosine monophosphate, PKG cGMP-dependent protein kinase, $H D L$ high-density lipoprotein, VLDL very low-density lipoprotein, IDL intermediate-density lipoprotein, LDL low-density lipoprotein

\section{Volume factors, kidney function and link to inflammation}

In order to try to understand underlying metabolic processes explaining the observations above, associations between NT-proBNP and the plasma and urine metabolome were investigated. Several associated metabolites, e.g. uridine in plasma as well as adenosine or 1-methylimidazole in urine point towards the vasodilatory role of BNP. For instance, uridine can induce vasodilation by activation of purinergic $\mathrm{P} 2 \mathrm{Y}$ receptors leading to release of nitric oxide, a well-known vasodilator [46]. The metabolite GPC is an abundant osmolyte in renal medulla cells and was inversely associated with NT-proBNP in plasma. Similarly, betaine acts also as osmolyte and accumulates together with GPC, sorbitol, and inositol to sufficiently counterbalance osmolality [47]. The inverse association of plasma GPC as well as urinary betaine with NT-proBNP appears to be a physiologic consequence of BNP signaling with respect to its role in diuresis.

Despite controlling for kidney function by means of the eGFR as surrogate marker, two metabolites in urine previously reported as surrogates for glomerular 
filtration in plasma [48], namely pseudouridine and C-mannosyltryptophan, were positively associated with NT-proBNP in linear regression analyses.

In previous work we identified urinary C-mannosyltryptophan as important predictor of an advanced inflammatory state in almost the same individuals as in the present investigation [49]. In this context the positive association of NT-proBNP with urinary neopterin might be of particular interest. Neopterin belongs to the group of pteridines and is derived from guanosine triphosphate in activated monocytes, macrophages, dendritic cells and endothelial cells, but also to a lesser extent in renal epithelial cells. Typically, neopterin is considered a marker of enhanced immune activation [50]. Neopterin in its unchanged form is excreted via the kidney and its levels correlate with the extent of disease or infection [51]. In addition, Grammer et al. identified NT-proBNP and also the eGFR as strongest predictors for neopterin serum concentration [50] linking neopterin to kidney function. Interestingly, also circulating adiponectin seems to be related with neopterin concentrations. Accordingly, a small observational study has observed that individuals with low HDL-cholesterol are characterized by low circulating adiponectin and high neopterin compared to individuals with high HDL-cholesterol This was interpreted as inflammatory phenotype in individuals with low HDLcholesterol [52] linking adiponectin also to inflammation.

Estimation of the glomerular filtration rate using serum creatinine suffers from a number of drawbacks, e.g. difference in muscle mass affecting creatinine release, so we could not rule out residual confounding via capturing the true glomerular filtration rate of the participants. However, the metabolite signature comprising urine levels of pseudouridine, C-mannosyltryptophan and neopterin links serum NT-proBNP to impaired kidney function rather than cardiac dysfunction.

\section{Steroidogenesis in the adrenal glands}

A cluster of adrenal-derived steroids and putative metabolites thereof (Fig. 2) was inversely associated with NTproBNP. It has been shown by Liang and coworkers in primary human adrenocortical cells, that BNP inhibited gene expression related to the angiotensin II induced steroidogenesis, including the de novo synthesis of cholesterol and other steps from cholesterol supply till steroid formation, namely cortisol, in mitochondria [53]. In line with this, NT-proBNP associated urinary cortisol metabolites indicate a potential shift in cortisol metabolism favoring activation or diminished degradation, as 11-ketoetiocholanolone glucuronide and the unknown $\mathrm{X}-12844$, recently identified as tetrahydrocortisone glucuronide [54], were inversely associated with NTproBNP. In line with the described inhibition of BNP on angiotensin II induced steroidogenesis, the strong inverse association with NT-proBNP may be a consequence of affected cortisol synthesis. Of note, adrenal glands are embedded into periadrenal adipose tissue and adiponectin receptors are expressed in human adrenal cortex [55]. Moreover, adiponectin itself was reported to affect adrenal steroidogenesis [56]. Thus, the observed associations with cortisol metabolites may implicate again that adiponectin may play a role in the observed associations. No association with plasma cortisol became obvious emphasizing local influence on cortisol metabolism as potential mechanism.

\section{Natriuretic peptides and energy consumption}

A prominent molecular signature related to NT-proBNP was a diminished urinary excretion of BCAAs and related intermediates. In line with the fact that obese individuals have lower levels of NT-proBNP independent of cardiac health [10], elevated circulating BCAA concentrations were found to be associated with obesity and insulin resistance, and have been linked to metabolic syndrome and CVD [57]. In regard of the prominent role of skeletal muscle as well as adipose tissue in BCAA catabolism [58], starting with the conversion of BCAA into their respective $\alpha$-keto acids within mitochondria, it might be conceivable that an inverse association of BCAA metabolites in urine with NT-proBNP is also related to BNP-signaling dependent increase in mitochondrial biogenesis. BNP-signaling induces mitochondrial biogenesis in skeletal muscles and enhances mitochondrial capacity of $\beta$-oxidation [59]. An increase in the number of mitochondria would in turn improve degradation of BCAAs and hence limit their urinary excretion. Notably, also adiponectin has been reported to induce mitochondrial biogenesis in skeletal muscle [60] posing again a relation between BNP and this adipokine. In this context, adiponectin has been shown to sequentially stimulate activity of the AMP-activated protein kinase (AMPK), p38 mitogen-activated protein kinase (MAPK) and peroxisome proliferator-activated receptor (PPAR) $\alpha$ thereby enhancing fatty acid oxidation in skeletal muscle [61].

Furthermore, natriuretic peptides have potent lipolytic effects in humans as observed upon intravenous infusion as well as subcutaneous infusion into abdominal adipose tissue [7, 62]. In line with this, NPR-A activation in adipocytes increased energy expenditure and heat production (thermogenesis) in mice $[62,63]$. Activation of brown adipose tissue is similarly inducible by cold-exposure. It has to be noted, that activation of brown adipose tissue by cold-exposure enhanced the triglyceride clearance from circulation, while HDL-cholesterol abundance increased and was sufficient to correct hyperlipidemia [64]. As natriuretic peptide signaling and cold-exposure 
seem to share effects on lipolysis and thermogenesis, it may be conceivable that also changes in the HDL metabolism, as observed in rodents and lean but not obese humans upon cold-exposure [65], may be among these shared effects providing a further possible explanation for the observed associations.

\section{Strengths and limitations}

The major strength of our study is the comprehensive profiling of the metabolome content of plasma and urine samples using the complementary techniques MS and ${ }^{1} \mathrm{H}-\mathrm{NMR}$ allowing us to screen for potential molecular signatures of alterations in NT-proBNP on a large-scale. The presence of only few individuals with self-reported HF minimized confounding of the associations by cardiac dysfunction but also limits our observations with respect to clinical application in this context. Nevertheless, the replication of the relation with a favorable lipid profile in an independent population strongly argues to augment the role of (NT-pro)BNP (and circulating adiponectin) concentrations in relation to cardiac dysfunction.

\section{Conclusion}

Considering the controversies that exist with respect to the paradox of low NT-proBNP in obese individuals and the known observation of increased NT-proBNP accompanying cardiac dysfunction for which obesity is a major risk factor, it can be noted from the present study that both observations are likely independent of each other. The present study demonstrates that NT-proBNP is positively associated with a beneficial lipoprotein profile covering almost the whole density gradient. As HF patients were almost absent in the present study population, the majority of the observed associations with small molecules likely reflect the physiological consequences of BNP signaling. Overall, the current data suggest a mechanism that, in absence of severe cardiac dysfunction, links higher NT-proBNP concentrations to a more favorable lipoprotein profile involving increased adiponectin release from adipose tissue. Future studies on this issue may further elucidate the underlying molecular mechanisms.

\section{Additional file}

Additional file 1. Detailed methods description and supporting results. Population characteristics for the replication population SHIP-2 and the results for the in-depth analysis of the lipoprotein profile are presented. Furthermore, the results of the sensitivity analysis on the influence of lipid lowering drugs on the lipoprotein profile is given.

\section{Abbreviations}

NT-proBNP: N-terminal fragment of the prohormone of brain natriuretic peptide; HF: heart failure; CVD: cardiovascular disease; ${ }^{1} \mathrm{H}-\mathrm{NMR}$ : proton nuclear magnetic resonance; MS: mass spectrometry; SHIP: Study of Health in Pomerania; WC: waist circumference; HDL: high-density lipoprotein; LDL: low-density lipoprotein; ALT: alanine amino transferase; eGFR: estimated glomerular filtration rate; EF: ejection fraction; VLDL: very-low-density lipoprotein; Apo: apolipoprotein; FDR: false discovery rate; GGM: Gaussian graphical modelling; IDL: intermediate-density lipoprotein; GPC: glycerophosphocholine; PC aa XX:Y: diacyl phosphatidylcholine; lysoPC: lysophosphatidylcholine; PVAT: perivascular adipose tissue; PKG: CGMP-dependent protein kinase; NPR-A: natriuretic peptide receptor A; T2DM: type 2 diabetes mellitus.

\section{Authors' contributions \\ AM and MP interpreted the data and wrote the manuscript. NF and MP designed the study protocol and analyzed the data. KB, AA and JA acquired spectroscopic and spectrometric data. MB and MD analyzed echocardio- graphic data and assisted in interpretation of results. HV provided SHIP data. GK and SZ assisted in data interpretation. NF and MN provided laboratory data and supervised the project. All authors read and approved the final manuscript.}

\section{Author details \\ ${ }^{1}$ Institute of Clinical Chemistry and Laboratory Medicine, University Medicine Greifswald, Ferdinand-Sauerbruch-Str, 17475 Greifswald, Germany. ${ }^{2}$ German Center for Cardiovascular Research (DZHK e.V.), Partner Site Greifswald, Greif- swald, Germany. ${ }^{3}$ Department of Internal Medicine B, University Medicine Greifswald, Greifswald, Germany. ${ }^{4}$ Institute of Bioinformatics and Systems Biology, Helmholtz Zentrum München, Neuherberg, Germany. ${ }^{5}$ Institute of Experimental Genetics, Genome Analysis Center, Helmholtz Zentrum München, Neuherberg, Germany. ${ }^{6}$ Lehrstuhl für Experimentelle Genetik, Technische Universität München, Freising-Weihenstephan, Germany. ${ }^{7}$ DZD (German Center for Diabetes Research), München-Neuherberg, Germany. ${ }^{8}$ Institute for Community Medicine, University Medicine Greifswald, Greif- swald 17475, Germany. ${ }^{9}$ DZD (German Center for Diabetes Research), Site Greifswald, Greifswald 17475, Germany.}

\section{Acknowledgements}

Not applicable.

\section{Competing interests}

The authors declare that they have no competing interests.

\section{Availability of data and materials}

SHIP data are publically available for scientific and quality control purposes. The informed consent obtained from the participants of the SHIP study does not cover data storage in public databases due to confidentially reasons. Data usage can be applied for via http://www.community-medicine.de.

\section{Consent for publication}

Not applicable.

\section{Ethics approval and consent to participate}

The study was approved by the local ethics committee and conformed to the principles of the declaration of Helsinki. All participants gave written informed consent before taking part in the study.

\section{Funding}

This work was funded by Grants from the German Federal Ministry of Education and Research (BMBF, Grants 01ZZ0403, 01ZZ0103, 01 Gl0883, AtheroSysMed 03IS2061B), the Ministry for Education, Research and Cultural Affairs, as well as the Ministry of Social Affairs of the Federal State of Mecklenburg-West Pomerania. This work is also part of the research project Greifswald Approach to Individualized Medicine (GANI_MED). The GANI_MED consortium is funded by the Federal Ministry of Education and Research and the Ministry of Cultural Affairs of the Federal State of Mecklenburg-West Pomerania (03IS2061A).

\section{Publisher's Note}

Springer Nature remains neutral with regard to jurisdictional claims in published maps and institutional affiliations.

Received: 10 July 2018 Accepted: 23 August 2018

Published online: 28 August 2018 


\section{References}

1. Ponikowski P, Voors AA, Anker SD, Bueno H, Cleland JG, Coats AJ, et al. 2016 ESC Guidelines for the diagnosis and treatment of acute and chronic heart failure: the Task Force for the diagnosis and treatment of acute and chronic heart failure of the European Society of Cardiology (ESC) Developed with the special contribution of the Heart Failure Association (HFA) of the ESC. Eur Heart J. 2016:37(27):2129-200.

2. Calzetta L, Orlandi A, Page C, Rogliani P, Rinaldi B, Rosano G, et al. Brain natriuretic peptide: much more than a biomarker. Int J Cardiol. 2016;221:1031-8

3. Kalra PR, Anker SD, Coats AJ. Water and sodium regulation in chronic heart failure: the role of natriuretic peptides and vasopressin. Cardiovasc Res. 2001;51(3):495-509.

4. Pandey KN. Biology of natriuretic peptides and their receptors. Peptides. 2005;26(6):901-32.

5. Jacob M, Saller T, Chappell D, Rehm M, Welsch U, Becker BF. Physiological levels of A-, B- and C-type natriuretic peptide shed the endothelial glycocalyx and enhance vascular permeability. Basic Res Cardiol. 2013;108(3):347.

6. Sengenes C, Berlan M, De Glisezinski I, Lafontan M, Galitzky J. Natriuretic peptides: a new lipolytic pathway in human adipocytes. FASEB J. 2000;14(10):1345-51.

7. Gruden G, Landi A, Bruno G. Natriuretic peptides, heart, and adipose tissue: new findings and future developments for diabetes research. Diabetes Care. 2014:37(11):2899-908.

8. Neeland IJ, Winders BR, Ayers CR, Das SR, Chang AY, Berry JD, et al. Higher natriuretic peptide levels associate with a favorable adipose tissue distribution profile. J Am Coll Cardiol. 2013;62(8):752-60.

9. Clerico A, Giannoni A, Vittorini S, Emdin M. The paradox of low BNP levels in obesity. Heart Fail Rev. 2012;17(1):81-96.

10. Wang TJ, Larson MG, Levy D, Benjamin EJ, Leip EP, Wilson PW, et al. Impact of obesity on plasma natriuretic peptide levels. Circulation. 2004;109(5):594-600.

11. He WT, Mori M, Yu XF, Kanda T. Higher BNP levels within physiological range correlate with beneficial nonfasting lipid profiles in the elderly: a cross-sectional study. Lipids Health Dis. 2016;15:3.

12. Sanchez OA, Duprez DA, Bahrami H, Daniels LB, Folsom AR, Lima JA, et al. The associations between metabolic variables and NT-proBNP are blunted at pathological ranges: the Multi-Ethnic Study of Atherosclerosis. Metabolism. 2014:63(4):475-83.

13. Sanchez OA, Duprez DA, Daniels LB, Maisel AS, Otvos JD, Peralta $\mathrm{CA}$, et al. The association between $\mathrm{N}$-terminal pro B-type natriuretic peptide and lipoprotein particle concentration plateaus at higher N-terminal pro B-type natriuretic peptide values: Multi-Ethnic Study on Atherosclerosis. Metabolism. 2015;64(8):857-61.

14. Nelson RH. Hyperlipidemia as a risk factor for cardiovascular disease. Prim Care. 2013:40(1):195-211.

15. Olsen MH, Hansen TW, Christensen MK, Gustafsson F, Rasmussen S, Wachtell K, et al. N-terminal pro brain natriuretic peptide is inversely related to metabolic cardiovascular risk factors and the metabolic syndrome. Hypertension. 2005:46(4):660-6.

16. Spannella F, Giulietti F, Cocci G, Landi L, Borioni E, Lombardi FE, et al. $\mathrm{N}$-terminal pro B-Type natriuretic peptide is inversely correlated with low density lipoprotein cholesterol in the very elderly. Nutr Metab Cardiovasc Dis. 2018:28(6):629-35.

17. Brutsaert EF, Biggs ML, Delaney JA, Djousse L, Gottdiener JS, Ix JH, et al. Longitudinal assessment of $\mathrm{N}$-terminal pro-B-type natriuretic peptide and risk of diabetes in older adults: the cardiovascular health study. Metabolism. 2016;65(10):1489-97.

18. Tanaka A, Yoshida H, Kawaguchi A, Oyama Jl, Kotooka N, Toyoda S, et al. $\mathrm{N}$-terminal pro-brain natriuretic peptide and associated factors in the general working population: a baseline survey of the Uranosaki cohort study. Sci Rep. 2017;7(1):5810.

19. Volzke H, Alte $\mathrm{D}$, Schmidt CO, Radke $\mathrm{D}$, Lorbeer $\mathrm{R}$, Friedrich $\mathrm{N}$, et al. Cohort profile: the study of health in Pomerania. Int J Epidemiol. 2011:40(2):294-307.

20. Inker LA, Schmid CH, Tighiouart H, Eckfeldt JH, Feldman HI, Greene T, et al. Estimating glomerular filtration rate from serum creatinine and cystatin C. N Engl J Med. 2012;367(1):20-9.
21. Volzke H, Haring R, Lorbeer R, Wallaschofski H, Reffelmann T, Empen K, et al. Heart valve sclerosis predicts all-cause and cardiovascular mortality. Atherosclerosis. 2010:209(2):606-10.

22. Schiller NB, Shah PM, Crawford M, DeMaria A, Devereux R, Feigenbaum $\mathrm{H}$, et al. Recommendations for quantitation of the left ventricle by twodimensional echocardiography. American society of echocardiography committee on standards, subcommittee on quantitation of two-dimensional echocardiograms. J Am Soc Echocardiogr. 1989;2(5):358-67.

23. Lang RM, Badano LP, Mor-Avi V, Afilalo J, Armstrong A, Ernande L, et al. Recommendations for cardiac chamber quantification by echocardiography in adults: an update from the American society of echocardiography and the European association of cardiovascular imaging. Eur Heart J Cardiovasc Imaging. 2015;16(3):233-70.

24. Knacke H, Pietzner M, Do KT, Romisch-MargI W, Kastenmuller G, Volker U, et al. Metabolic fingerprints of circulating IGF-1 and the IGF-1/ IGFBP-3 ratio: a multifluid metabolomics study. J Clin Endocrinol Metab. 2016;101(12):4730-42.

25. Pietzner M, Homuth G, Budde K, Lehmphul I, Volker U, Volzke H, et al. Urine Metabolomics by (1)H-NMR spectroscopy indicates associations between serum 3,5-T2 concentrations and intermediary metabolism in euthyroid humans. Eur Thyroid J. 2015;4(Suppl 1):92-100.

26. Stone CJ, Koo C-Y. Additive splines in statistics. Proc Stat Comp Sect Am Stat Assoc. 1985;27:45-8.

27. Krumsiek J, Suhre K, Illig T, Adamski J, Theis FJ. Gaussian graphical modeling reconstructs pathway reactions from high-throughput metabolomics data. BMC Syst Biol. 2011;5:21.

28. Weber M, Hamm C. Role of B-type natriuretic peptide (BNP) and NTproBNP in clinical routine. Heart. 2006;92(6):843-9.

29. Ebbert JO, Jensen MD. Fat depots, free fatty acids, and dyslipidemia. Nutrients. 2013;5(2):498-508.

30. Takeuchi H, Sata M. The relationship among brain natriuretic peptide (BNP), cholesterol and lipoprotein. Heart Asia. 2012;4(1):11-5.

31. Ohara T, Kim J, Asakura M, Asanuma H, Nakatani S, Hashimura K et al. Plasma adiponectin is associated with plasma brain natriuretic peptide and cardiac function in healthy subjects. Hypertens Res. 2008:31(5):825-31.

32. Fiaschi T, Magherini F, Gamberi T, Modesti PA, Modesti A. Adiponectin as a tissue regenerating hormone: more than a metabolic function. Cell Mol Life Sci. 2014;71(10):1917-25.

33. Jung YR, Lee JH, Sohn KC, Lee Y, Seo YJ, Kim CD, et al. Adiponectin signaling regulates lipid production in human sebocytes. PLOS ONE. 2017:12(1):e0169824.

34. Withers SB, Bussey CE, Saxton SN, Melrose HM, Watkins AE, Heagerty AM. Mechanisms of adiponectin-associated perivascular function in vascular disease. Arterioscler Thromb Vasc Biol. 2014;34(8):1637-42.

35. Tsukamoto O, Fujita M, Kato M, Yamazaki S, Asano Y, Ogai A, et al. Natriuretic peptides enhance the production of adiponectin in human adipocytes and in patients with chronic heart failure. J Am Coll Cardiol. 2009:53(22):2070-7.

36. Schneider JG, von Eynatten M, Schiekofer S, Nawroth PP, Dugi KA. Low plasma adiponectin levels are associated with increased hepatic lipase activity in vivo. Diabetes Care. 2005;28(9):2181-6.

37. Qiao L, Zou C, van der Westhuyzen DR, Shao J. Adiponectin reduces plasma triglyceride by increasing VLDL triglyceride catabolism. Diabetes. 2008:57(7):1824-33.

38. Christou GA, Kiortsis DN. Adiponectin and lipoprotein metabolism. Obes Rev. 2013;14(12):939-49.

39. Lugrin J, Rosenblatt-Velin N, Parapanov R, Liaudet L. The role of oxidative stress during inflammatory processes. Biol Chem. 2014;395(2):203-30.

40. Winsz-Szczotka K, Kuznik-Trocha K, Komosinska-Vassev K, Kucharz E, Kotulska A, Olczyk K. Relationship between adiponectin, leptin, IGF-1 and total lipid peroxides plasma concentrations in patients with systemic sclerosis: possible role in disease development. Int J Rheum Dis. 2016:19(7):706-14.

41. Antonopoulos AS, Margaritis M, Coutinho P, Shirodaria C, Psarros C, Herdman $\mathrm{L}$, et al. Adiponectin as a link between type 2 diabetes and vascular NADPH oxidase activity in the human arterial wall: the regulatory role of perivascular adipose tissue. Diabetes. 2015;64(6):2207-19. 
42. Hansen CS, Vistisen D, Jorgensen ME, Witte DR, Brunner EJ, Tabak AG, et al. Adiponectin, biomarkers of inflammation and changes in cardiac autonomic function: whitehall II study. Cardiovasc Diabetol. 2017;16(1):153.

43. Kubota M, Yoneda M, Maeda N, Ohno H, Oki K, Funahashi T, et al. Westernization of lifestyle affects quantitative and qualitative changes in adiponectin. Cardiovasc Diabetol. 2017;16(1):83.

44. Ida S, Murata K, Betou K, Kobayashi C, Ishihara Y, Imataka K, et al. Effect of trelagliptin on vascular endothelial functions and serum adiponectin level in patients with type 2 diabetes: a preliminary single-arm prospective pilot study. Cardiovasc Diabetol. 2016;15(1):153.

45. Ortega Moreno L, Copetti M, Fontana A, De Bonis C, Salvemini L, Trischitta $\checkmark$, et al. Evidence of a causal relationship between high serum adiponectin levels and increased cardiovascular mortality rate in patients with type 2 diabetes. Cardiovasc Diabetol. 2016;15:17.

46. Wihlborg AK, Malmsjo M, Eyjolfsson A, Gustafsson R, Jacobson K, Erlinge D. Extracellular nucleotides induce vasodilatation in human arteries via prostaglandins, nitric oxide and endothelium-derived hyperpolarising factor. Br J Pharmacol. 2003;138(8):1451-8.

47. Gallazzini M, Burg MB. What's new about osmotic regulation of glycerophosphocholine. Physiology (Bethesda). 2009;24:245-9.

48. Sekula P, Goek ON, Quaye L, Barrios C, Levey AS, Romisch-MargI W, et al. A metabolome-wide association study of kidney function and disease in the general population. J Am Soc Nephrol. 2016;27(4):1175-88.

49. Pietzner M, Kaul A, Henning AK, Kastenmuller G, Artati A, Lerch MM, et al. Comprehensive metabolic profiling of chronic low-grade inflammation among generally healthy individuals. BMC Med. 2017;15(1):210.

50. Grammer TB, Fuchs D, Boehm BO, Winkelmann BR, Maerz W. Neopterin as a predictor of total and cardiovascular mortality in individuals undergoing angiography in the Ludwigshafen Risk and Cardiovascular Health study. Clin Chem. 2009;55(6):1135-46.

51. Fuchs D, Stahl-Hennig C, Gruber A, Murr C, Hunsmann G, Wachter H. Neopterin-its clinical use in urinalysis. Kidney Int Suppl. 1994;47:S8-11.

52. Holven KB, Retterstol K, Ueland T, Ulven SM, Nenseter MS, Sandvik M, et al. Subjects with low plasma HDL cholesterol levels are characterized by an inflammatory and oxidative phenotype. PLOS ONE. 2013;8(11):e78241.

53. Liang F, Kapoun AM, Lam A, Damm DL, Quan D, O'Connell M, et al. B-Type natriuretic peptide inhibited angiotensin II-stimulated cholesterol biosynthesis, cholesterol transfer, and steroidogenesis in primary human adrenocortical cells. Endocrinology. 2007;148(8):3722-9.
54. Long T, Hicks M, Yu HC, Biggs WH, Kirkness EF, Menni C, et al. Wholegenome sequencing identifies common-to-rare variants associated with human blood metabolites. Nat Genet. 2017:49(4):568-78.

55. Rossi GP, Sticchi D, Giuliani L, Bernante P, Zavattiero S, Pessina AC, et al. Adiponectin receptor expression in the human adrenal cortex and aldosterone-producing adenomas. Int J Mol Med. 2006;17(6):975-80.

56. Li P, Sun F, Cao HM, Ma QY, Pan CM, Ma JH, et al. Expression of adiponectin receptors in mouse adrenal glands and the adrenocortical Y-1 cell line: adiponectin regulates steroidogenesis. Biochem Biophys Res Commun. 2009;390(4):1208-13

57. Lynch CJ, Adams SH. Branched-chain amino acids in metabolic signalling and insulin resistance. Nat Rev Endocrinol. 2014;10(12):723-36.

58. Crown SB, Marze N, Antoniewicz MR. Catabolism of branched chain amino acids contributes significantly to synthesis of odd-chain and evenchain fatty acids in 3T3-L1 adipocytes. PLOS ONE. 2015;10(12):e0145850.

59. Moro C, Smith SR. Natriuretic peptides: new players in energy homeostasis. Diabetes. 2009;58(12):2726-8.

60. Qiao L, Kinney B, Yoo HS, Lee B, Schaack J, Shao J. Adiponectin increases skeletal muscle mitochondrial biogenesis by suppressing mitogenactivated protein kinase phosphatase-1. Diabetes. 2012;61(6):1463-70.

61. Yoon MJ, Lee GY, Chung JJ, Ahn YH, Hong SH, Kim JB. Adiponectin increases fatty acid oxidation in skeletal muscle cells by sequential activation of AMP-activated protein kinase, p38 mitogen-activated protein kinase, and peroxisome proliferator-activated receptor alpha. Diabetes. 2006;55(9):2562-70.

62. Coue M, Moro C. Natriuretic peptide control of energy balance and glucose homeostasis. Biochimie. 2016;124:84-91.

63. Bordicchia M, Liu D, Amri EZ, Ailhaud G, Dessi-Fulgheri P, Zhang C, et al. Cardiac natriuretic peptides act via p38 MAPK to induce the brown fat thermogenic program in mouse and human adipocytes. J Clin Invest. 2012;122(3):1022-36

64. Bartelt A, Bruns OT, Reimer R, Hohenberg H, Ittrich H, Peldschus K, et al. Brown adipose tissue activity controls triglyceride clearance. Nat Med. 2011;17(2):200-5

65. Bartelt A, John C, Schaltenberg N, Berbee JFP, Worthmann A, Cherradi $\mathrm{ML}$, et al. Thermogenic adipocytes promote HDL turnover and reverse cholesterol transport. Nat Commun. 2017;8:15010.
Ready to submit your research? Choose BMC and benefit from:

- fast, convenient online submission

- thorough peer review by experienced researchers in your field

- rapid publication on acceptance

- support for research data, including large and complex data types

- gold Open Access which fosters wider collaboration and increased citations

- maximum visibility for your research: over $100 \mathrm{M}$ website views per year

At BMC, research is always in progress.

Learn more biomedcentral.com/submissions 